\title{
Proteome-scale profiling reveals MAFF and MAFG as two novel key transcription factors involved in palmitic acid-induced umbilical vein endothelial cell apoptosis
}

\author{
Mangyuan Wang ${ }^{1,2,4}$, Fen Liư ${ }^{4}$ Binbin Fang ${ }^{4}$, Qiang Huo ${ }^{2^{*}}$ and Yining Yang ${ }^{3,4^{*}}$ [D
}

\begin{abstract}
Background: Vascular endothelial cell apoptosis is the leading risk factor of atherosclerosis (AS). The purpose of our study was to use a new generation high-throughput transcription factor (TF) detection method to identify novel key TFs in vascular endothelial cell apoptosis induced by palmitic acid (PA).

Methods: Human umbilical vein endothelial cells (HUVECs) were treated with 0, 300, or 500 MM PA. Candidate TFs in the three groups were identified by differential expression, pathway enrichment, Western Blot (WB), and RT-qPCR analyses. Apoptosis was assessed by fluorescence-activated cell sorting (FACS) using FITC-annexin V and propidium iodide staining.

Results: We established a HUVEC apoptosis model to simulate the process of atherosclerosis onset and identified 51 significant TFs. of the 51 TFs, v-maf musculoaponeurotic fibrosarcoma oncogene family protein G (MAFG) and v-maf musculoaponeurotic fibrosarcoma oncogene family protein $F$ (MAFF), were matched to known AS signalling pathways and were validated by WB and RT-qPCR analyses in our study. Overexpression of MAFG or MAFF in HUVECS significantly inhibited PA-induced early apoptosis.
\end{abstract}

Conclusions: We identified MAFF and MAFG as novel key TFs in vascular endothelial cell apoptosis.

Keywords: Apoptosis, Atherosclerosis, Endothelial cell, HUVEC, Palmitic acid, Transcription factor

\section{Background}

Atherosclerosis (AS) is a common vascular disease characterized by atherosclerotic plaque formation, which is initiated by endothelial cell apoptosis followed by lipid deposition and foam cell formation [1]. AS is the leading

*Correspondence: huogiang2019@163.com; yangyn5126@163.com ${ }^{2}$ Department of Cardiac Surgery, The First Affiliated Hospital of Xinjiang Medical University, 137, Liyushan Road, Xin Shi District, Urumqi 830054, People's Republic of China

${ }^{3}$ Department of Cardiology, The First Affiliated Hospital of Xinjiang

Medical University, 137, Liyushan Road, Xin Shi District, Urumqi 830054,

People's Republic of China

Full list of author information is available at the end of the article cause of cardiac infarction, arterial aneurysm, stroke, and the severe associated complications [2].

Vascular endothelial cell apoptosis is the initial pathogenetic process underlying atherosclerotic plaque formation, and it is the leading risk factor for atherosclerotic plaque formation [1]. To interfere effectively in the early stage of AS and to predict the atherosclerotic plaque formation risk, we focused on identifying the key transcription factors (TFs) in this initial process of AS formation. Human umbilical vein endothelial cells (HUVECs) are cells that recapitulate the characteristics of arterial endothelial cells in vitro. HUVECs with apoptosis induced by $24 \mathrm{~h}$ of stimulation with palmitic acid (PA) 
are a mature cell model of AS [3]. Numerous TFs and signalling pathways, as well as changes in gene expression levels, are likely to be involved in HUVEC apoptosis [4].

TF response element (TFRE) profiling is a new-generation high-throughput TF detection method that allows qualitative and semiquantitative screening of TFs on the proteome scale in specific cells or tissues using nanoliquid chromatography/tandem mass spectrometry (LCMS) analysis [5]. TFRE profiling provides information that directly reflects changes in TF protein levels as well as the activation state of the TF population.

Although previous studies have reported some TFs that are key players in HUVEC apoptosis [6], high-throughput TF profiling in HUVEC apoptosis models has not yet been reported.

In this study, to identify key TFs involved in HUVEC apoptosis, we conducted TFRE profiling using three experimental groups of HUVECs treated with increasing concentrations of PA to induce apoptosis, and followed with cellular functional validation experiments.

\section{Methods}

Experimental groups, workflow, and study design of TFRE profiling

HUVECs were grouped as follows: control group; HUVECs treated with $0 \mu \mathrm{M}$ PA; group 1, HUVECs stimulated with $300 \mu \mathrm{M} \mathrm{PA}$; and experimental group 2, HUVECs stimulated with $500 \mu \mathrm{M}$ PA. All three groups were treated with PA for $24 \mathrm{~h}$, and flow cytometry was performed to test the early apoptosis rates among the three groups three times to determine whether the HUVEC apoptosis model to simulate the onset process of atherosclerosis was established. Then, TFRE profiling experiments were performed three times.

\section{HUVEC cultivation}

Primary HUVECs were purchased from COBIOER company (Nanjing, China), and the designated type was primary cells from PromoCell. The cells were checked to ensure that they were free of contamination, and they were used from a low-passage stock. We then cultured the cells with BEGM BulletKit Medium (LONZA, catalogue no. CC-3170). HUVECs were seeded into 18 culture flasks $\left(25 \mathrm{~cm}^{2}\right.$ in size), of which three flasks were used for each of the three experimental groups. The cells grew and adhered to the flask walls, and the cell density in each flask was monitored. We added PA to the cell culture when the density was $1 \times 10^{5}$ cells $/ \mathrm{cm}^{2}$ at 3 days after cell passage. For each group, the six flasks were then combined into three sets of three flasks, which were considered one replicate; the experiment was therefore conducted with three replicates.
SV40T-transformed HUVECs were purchased from ProCell company (catalogue no. CL-0675, Wuhan, China). SV40T-transformed HUVECs were cultured in DMEM-H (Dulbecco's modified Eagle's medium $+10 \%$ FBS $+1 \%$ NEAA) in an incubator at $37{ }^{\circ} \mathrm{C}$ with $95 \%$ humidity and $5 \%$ carbon dioxide. We passaged the cells every 3 days.

\section{Nuclear protein extraction}

A Pierce NE-PER kit (Thermo Scientific, USA) was used to extract the nuclear proteins of the HUVECs. The concentration of nuclear protein was determined by spectrophotometry, and then the volume of nuclear extract was adjusted to guarantee the comparability of protein input among the three groups.

Enrichment of TFs in nuclear protein extracts using catTFRE The catTFRE DNA chains were amplified from a plasmid containing catTFRE with biotin-labelled primers (Sigma) via PCR and were bound to Dynabeads M-280 Streptavidin (Invitrogen, USA). The catTFRE-bead complex was then mixed with the nuclear protein extracts and incubated at $4{ }^{\circ} \mathrm{C}$ for $2 \mathrm{~h}$ to enrich the transcription factors in the nuclear protein extracts. The detailed TFRE procedures are described in our previous study [7].

\section{Nano-LC-MS/MS analysis for protein identification and label-free quantification}

We dissolved dry tryptic peptides in $1 \%$ acetonitrile containing $0.1 \%$ methanol. Next, we separated one-third of the volume of the peptides on a C18 column, and the peptide samples were analysed on an EASY-nLC 1000 LC system (Thermo Fisher Scientific, Waltham, MA) coupled with Orbitrap Fusion mass spectrometry (Thermo Fisher Scientific, Waltham, MA). MS raw peptide data generated by LC-MS/MS were searched against the UniProt human proteome database (version 2019-03-07, 20, 404 sequences) using MaxQuant (version 1.6.5.0) software (Additional file 8). Finally, we used Proteome Discoverer 1.4 to select TFs from the identified proteins.

\section{Selection of candidates for key TFs in HUVEC apoptosis}

The fraction of total (FOT)-adjusted peak areas were compared among the three groups, and heatmap and volcano plots were drawn based on TF showing variations with both t-test $P$ value $<0.05$ and fold change $>2$. Then, a relational network among these TFs was generated to find the central TFs. Next, we selected differential TFs showing the same variation trends among the three experimental groups. Downstream gene pathway enrichment analysis of the differential TFs with the same variation trends was conducted to select TFs matching known signalling pathways in AS, which were selected 
as candidate TFs involved in HUVEC apoptosis. Then, we excluded known key TFs involved in AS in other previous studies, and the remaining TFs were selected as candidate novel TFs involved in HUVEC apoptosis after Western Blot (WB) and real-time quantitative PCR (RTqPCR) verification.

\section{Western blot (WB) analysis}

Total proteins were extracted from HUVECs in the 3 groups. The proteins were separated by SDS-PAGE and transferred to PVDF membranes. Membranes were incubated with the primary antibodies (anti-MAFG, Abcam, ab154318, 1:500; anti-MAFF, Abcam, ab183859, 1:500) overnight at $4{ }^{\circ} \mathrm{C}$ after blocking with $5 \%$ skim milk for $1 \mathrm{~h}$. Then, membranes were incubated with the secondary antibody (Abcam, ab205718) labelled with horseradish peroxidase. After incubation, we performed washing with TBST (Tris-buffered saline containing Tween 20). The immune complex on the membrane was then visualized by diaminobenzidine. The integrated density was determined by Image $\mathrm{Lab}^{\mathrm{TM}}$ software version 4.0 (Bio-Rad Laboratories, Inc., Hercules, CA, USA) and normalized to a percentage of the internal control $\beta$-actin. Images of original Western Blots were illustrated in Figure S3 (Additional file 3).

\section{Real time quantitative PCR (RT-qPCR)}

Total RNA was extracted from HUVECs with TRIzol reagent. cDNA was synthesized with a reverse transcription kit (QIAGEN, China). RT-qPCR was performed with Power SYBR Green PCR Master Mix (Applied Biosystems, U.S.A.) in a 7900 HT Fast Real-Time PCR system (Applied Biosystems, U.S.A.). The results were calculated with the $2^{-\Delta \Delta C t}$ method and normalized to a percentage of the internal control of $\beta$-actin. The primers used in the present study are listed in Table 1.

\section{MAFF or MAFG overexpression (OE) plasmid construction} and transfection of SV40T-transformed HUVECs

The plasmid pIRES2-EGFP containing human MAFF cDNA (pMAFF-IRES2-EGFP, Additional file 1: Figure S1) and human MAFG cDNA (pMAFG-IRES2-EGFP, Additional file 2: Figure S2) was constructed by TSINGKE BioTech (Beijing, China). The forward primer for MAFF was CTCGAGATGTCTGTGGATCC, and the reverse

Table 1 Primers using in RT-qPCR to validate the TFRE results

\begin{tabular}{lll}
\hline Molecules & Forward & Reverse \\
\hline MAFG & TCCAGGGTACTGACCTGCTC & GTTTCCTTTATTGGGGGTCG \\
MAFF & TGCCCAGGTCCCATTTCTC & GGCCCACGAAGGGAATGT \\
$\beta$-actin & CCTAGAAGCATTTGCGGTGG & GAGCTACGAGCTGCCTGACG \\
\hline
\end{tabular}

primer for MAFF was GAATTCCTAGGAGCAGGA. The forward primer for MAFG was CTCGAGATGACG ACCCCCA, and the reverse primer for MAFG was GAA TTCCTACGATCGGGC.

After passage for three generations, the cells were transfected for $48 \mathrm{~h}$ with MAFF or MAFG OE plasmid or $10 \mu \mathrm{g}$ of empty plasmid with $10 \mu \mathrm{l}$ of Lipofectamine 2000 Transfection Reagent (catalogue no.: 11668027, Thermo Fisher Scientific, USA) in each group. We used a fluorescence microscope to observe the GFP signal.

\section{RT-qPCR and WB validation of MAFF or MAFG OE}

RT-qPCR and WB validation of MAFF or MAFG OE was performed in four groups: the control group without plasmid transfection; empty plasmid transfection group; MAFF OE plasmid transfection group; and MAFG OE plasmid transfection group.

The detailed procedures for RT-qPCR and WB analysis are as above in Sects. Western blot (WB) analysis and Real time quantitative PCR (RT-qPCR). The primers used for validation of MAFF and MAFG OE are listed in Table 2.

\section{Cellular functional validation of MAFF and MAFG and flow cytometric analysis}

HUVECs were grouped into 4 experimental groups: the control group (no plasmid and no $\mathrm{OE}$ ), plasmid group (empty plasmid and no OE), MAFF OE group (MAFF OE plasmid and MAFF OE), and MAFG OE group (MAFG $\mathrm{OE}$ plasmid and MAFG $\mathrm{OE}$ ). The four groups were induced by $500 \mu \mathrm{M}$ PA for $24 \mathrm{~h}$.

HUVECs were harvested from culture plates using trypsin/EDTA and washed in PBS. Staining was performed for $30 \mathrm{~min}$ on ice with FITC-annexin V $(30 \mu \mathrm{g} /$ $\mathrm{ml}$ ) and propidium iodide (PI, $30 \mu \mathrm{g} / \mathrm{ml}$, BD Pharmingen, San Diego, CA, USA) in calcium-containing PBS. Fluorescence-activated cell sorting (FACS) analysis was performed immediately (Mindray BriCyte E6 FACS Calibur instrument). A total of $10^{5}$ cells/sample were counted, and the results were evaluated by using Cell Quest software (Becton Dickinson).

Table 2 Primers of RT-qPCR to validate the OE of MAFF and MAFG

\begin{tabular}{lll}
\hline Molecules & Forward & Reverse \\
\hline MAFG & AAGGCCTTGAAGGTGAAGCG & CCTTCTGCTTCTCCAGCTCC \\
MAFF & GAGGAGCTGCAGAAGCAGAA & TGACGATGGTGATGACGCTG \\
GAPDH & ACAGCAACAGGGTGGTGAC & TTTGAGGGTGCAGCGAACTT \\
\hline
\end{tabular}


(See figure on next page.)

Fig. 1 Establishment of the HUVEC apoptosis model to simulate the initiation process of atherosclerosis. Representative flow cytometric analysis showing the apoptosis of HUVECs in the control group $(\mathbf{a}, \mathbf{d}, \mathbf{g})$, group $1(\mathbf{b}, \mathbf{e}, \mathbf{h})$, and group $2(\mathbf{c}, \mathbf{f}, \mathbf{i})$. j Quantitative analysis of the increased early apoptosis percentage in HUVECs treated with various concentrations of PA $(n=3)$. Control group: $0 \mu M$ PA. Group 1: $300 \mu M$ PA. Group 2: $500 \mu M$ PA. ** indicates $0.001<P<0.01$

\section{Statistical analysis}

We used the fraction of total (FOT) value to adjust the peak area among groups, and we used KolmogorovSmirnov and Shapiro-Wilk tests to test the normality of continuous variables. We used unpaired t-tests to compare continuous variables with a normal distribution. We used the Mann-Whitney $U$ test to compare continuous variables with skewed distributions. A significant difference between two groups was represented by a twotailed $P<0.05$. SPSS (version: 20.0) was used to analyse data. GraphPad Prism (version: 6.01) and Adobe Illustrators were used to generate and compose the figures.

\section{Results}

Establishment of a HUVEC apoptosis model to simulate the onset process of atherosclerosis.

We stimulated HUVECs at $300 \mu \mathrm{M}$ and $500 \mu \mathrm{M}$, and flow cytometry was performed to observe the changes in the early apoptosis rate. We found that the early apoptosis rate increased significantly $(15.37 \% \pm 0.85 \%$ vs $42.17 \% \pm 7.14 \%, \mathrm{P}=0.022)$ as the $\mathrm{PA}$ concentration increased from $300 \mu \mathrm{M}$ in group 1 to $500 \mu \mathrm{M}$ in group 2 (Fig. 1).

\section{Identification of $51 \mathrm{TFs}$ showing significant differential expression $(P<0.05$ and fold change $>2)$}

In the three TFRE profiling experiments, we identified 173 TFs in total in the control group, group 1, and group 2 . Of these, 36 TFs showed significant differential expression with a t-test $P$ value $<0.05$ between group 1 and the control group, 34 TFs were significantly differentially expressed between group 2 and the control group, and 13 TFs were significantly differentially expressed between group 2 and group 1. All 51 significant TFs (Additional file 4: Table S1) are shown in Fig. 2a.

As shown in the volcano plots (Fig. 2b-d), 34 TFs (Additional file 5: Table S2) significantly differentially expressed between group 1 and the control group were identified and met the selection criteria of both $P$ value $<0.05$ and fold change $>2$ (Fig. $2 b$ ). Of these, 11 TFs were upregulated, shown as red dots in Fig. 2b, and 23 TFs were downregulated, shown as blue dots in Fig. $2 \mathrm{~b}$. Thirteen TFs (Additional file 6: Table S3) showing significantly different levels between group 2 and group 1 met the selection criteria (Fig. 2c), of which 8 TFs were upregulated (red dots) and 5 TFs were downregulated (blue dots). Finally, 33 TFs (Additional file 7: Table S4) showing significant differential expression between group 2 and the control group met the selection criteria (Fig. 2d); of these, 12 TFs were upregulated (red dots) and 21 TFs were downregulated (blue dots).

\section{Protein interaction network analysis and TFs with significant dose-dependent increased or decreased expression between the control group and group 1 and between group 1 and group 2}

Protein interaction network analysis (Fig. 3a) revealed that several TFs, including ATF3, JUN, JUNB, MAFG, MAFF, RELA, STAT1, and STAT6, were significantly differentially expressed and interconnected. Among the TFs showing differential expression between the control group and group 1 and between group 1 and group 2 (with dose dependence), 12 of the 51 significant TFsnamely ATF3, XBP1, STAT1, STAT6, AEBP1, MAFG, NFE2L1, CBFB, JUNB, NFAT5, HOXD8, and MAFFwere upregulated in group 1 compared with the control group and further upregulated in group 2 compared with group 1 (Fig. 3b, c), while 12-namely, HOXA5, ALX1, ELK3, RARB, ATF7, AHR, PBX2, MAX, FLI, ARID1A, JUN, and E2F4-were downregulated in group 1 compared with the control group and further downregulated in group 2 compared with group 1 (Fig. 3d, e). Therefore, the expression of these 12 upregulated TFs and 12 downregulated TFs was dose-dependent. Seven TFs overlapped between the significant TFs in the interaction network analysis and the TFs with dose-dependent significantly differential expression in the control group compared with group 1 and in group 1 compared with group 2: ATF3, JUN, JUNB, MAFG, MAFF, STAT1, and STAT6.

Pathway enrichment of TFs and matching to key signalling pathways of AS; WB and RT-qPCR validation

To identify which of the 7 TFs are correlated with known AS pathways to further narrow the range of the TFRE screening results for novel key TFs involved in AS initiation, we performed downstream pathway enrichment analysis of the 7 overlapping TFs (Fig. 4), and 4 TFs were matched to known key signalling pathways of ASnamely, the NFE2L2 (Nrf2)-ARE, NFKB, and MAPK signalling pathways. The analysis identified four TFs: JUN 

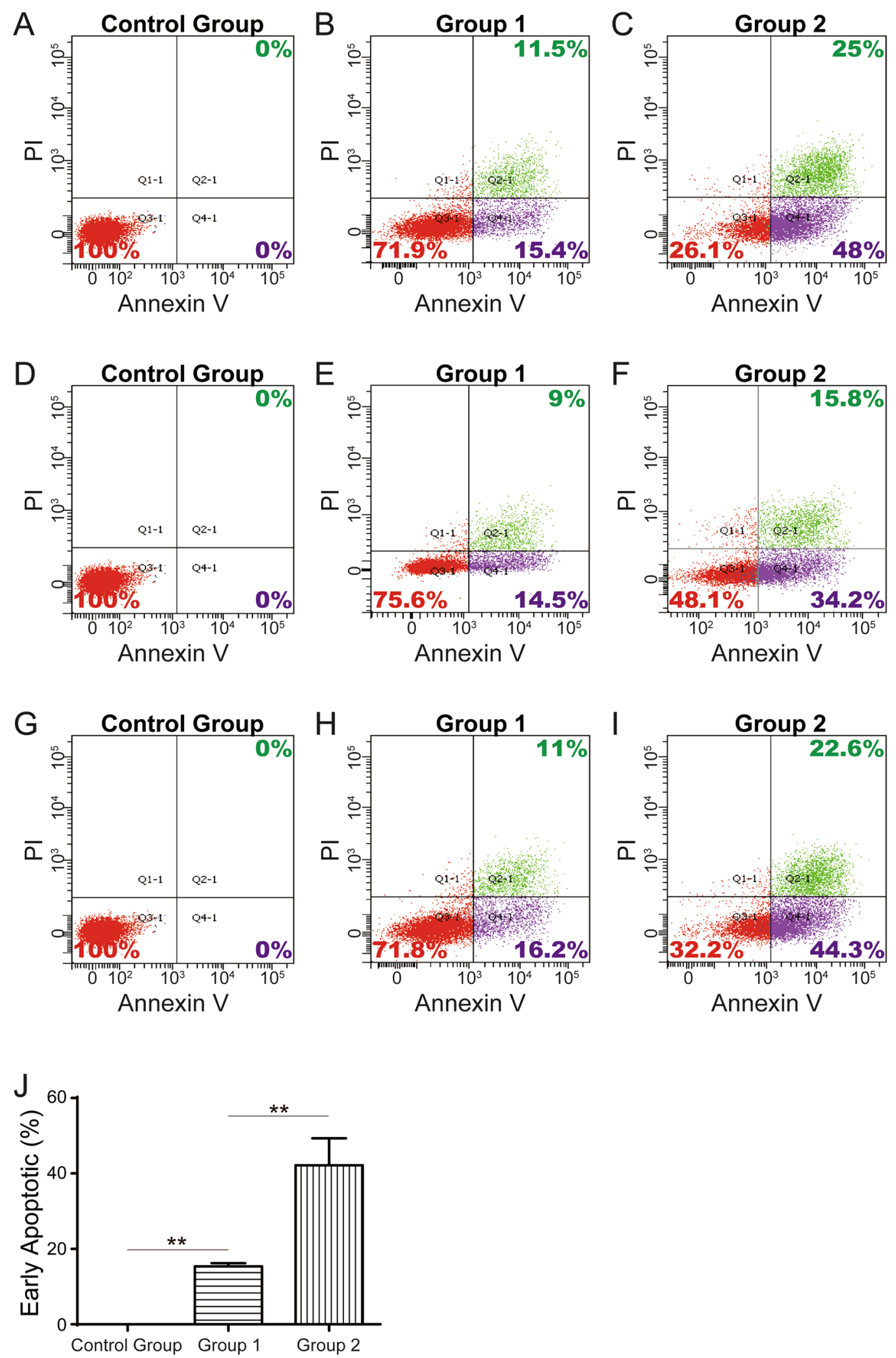

Fig. 1 (See legend on previous page.) 
A

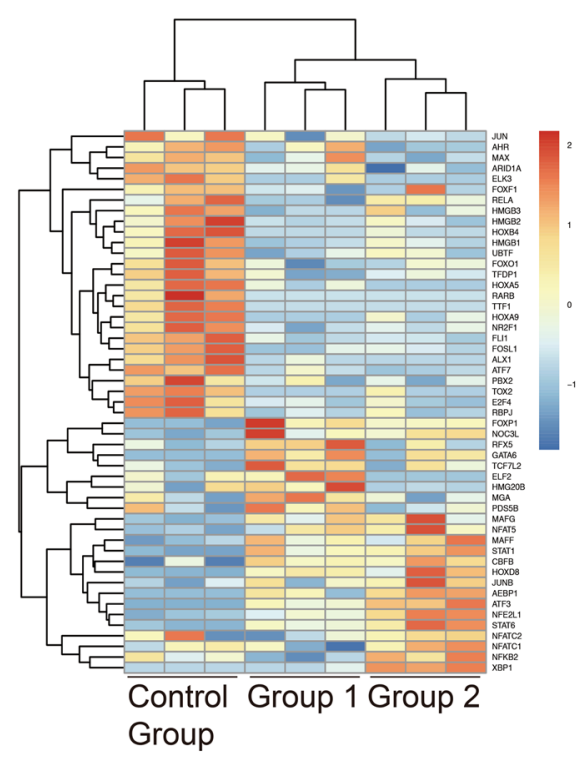

C

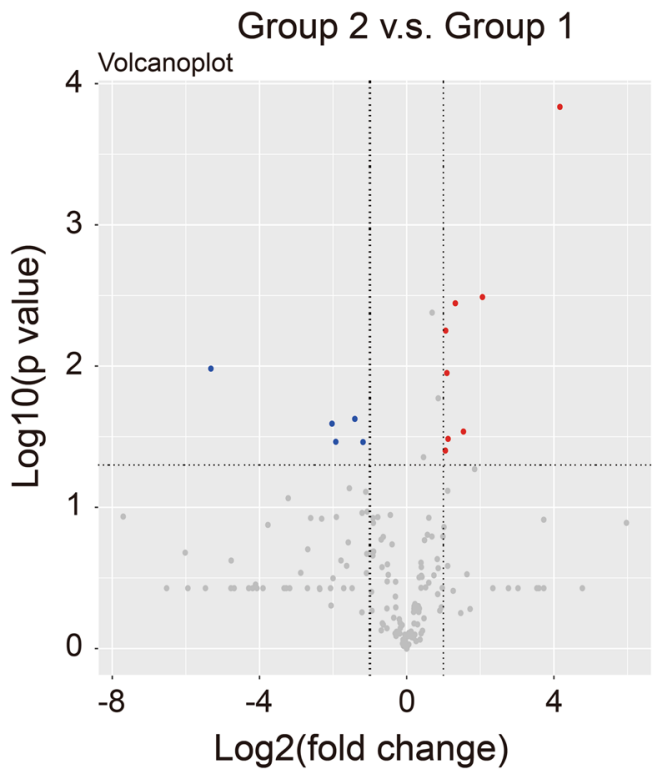

$\mathrm{B}$

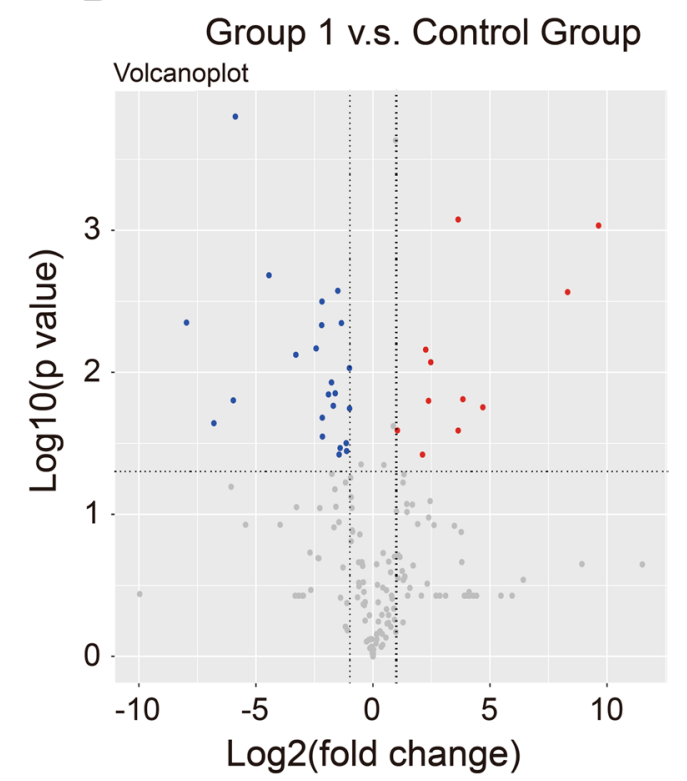

D

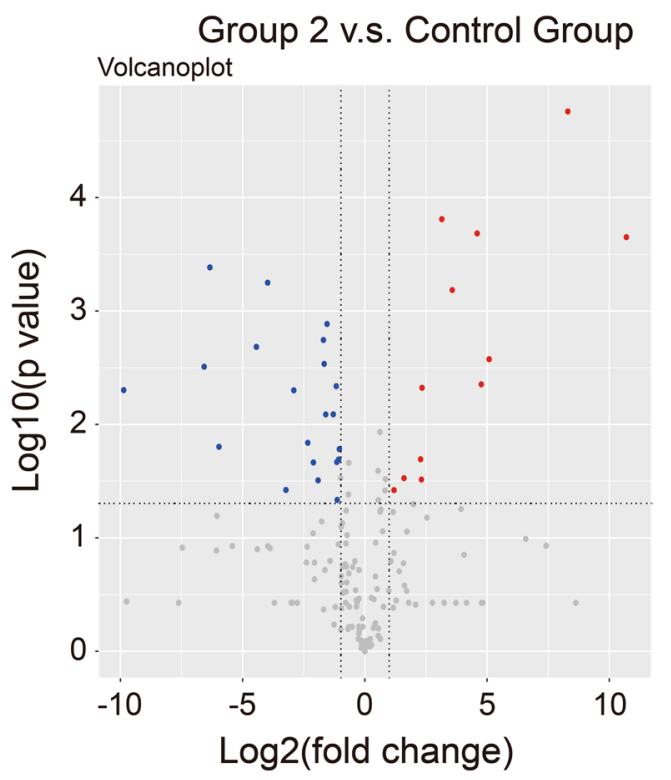

Fig. 2 Identification of 51 significant TFs with a t-test $P$ value $<0.05$ and fold change $>2$. a The heatmap shows 51 transcription factors (TFs) that showed significant differential expression among the three experimental groups. Blue and red represent low and high expression levels, respectively. $\mathbf{b}$ - $\mathbf{d}$ Each dot in the volcano plots shows one TF with both a fold change $>2$ and a t-test $P$ value $<0.05$. The red dots represent upregulated transcription factors and the blue dots represent downregulated transcription factors between two of the three groups

(AP1) (Fig. 4c), MAFF (Fig. 4e), MAFG (Fig. 4f), and STAT1 (Fig. 4g).

We identified four candidate TFs-JUN (AP1), MAFG, MAFF, and STAT1-in the previous "Protein interaction network analysis and TFs with significant dose-dependent increased or decreased expression between the control group and group 1 and between group 1 and group 2" section, and two of the four TFs, JUN and STAT1, were then found to be two known key TFs in AS in other former studies by searching articles $[8,9]$. Therefore, the remaining two novel candidate key TFs, MAFF and MAFG, were verified by performing 

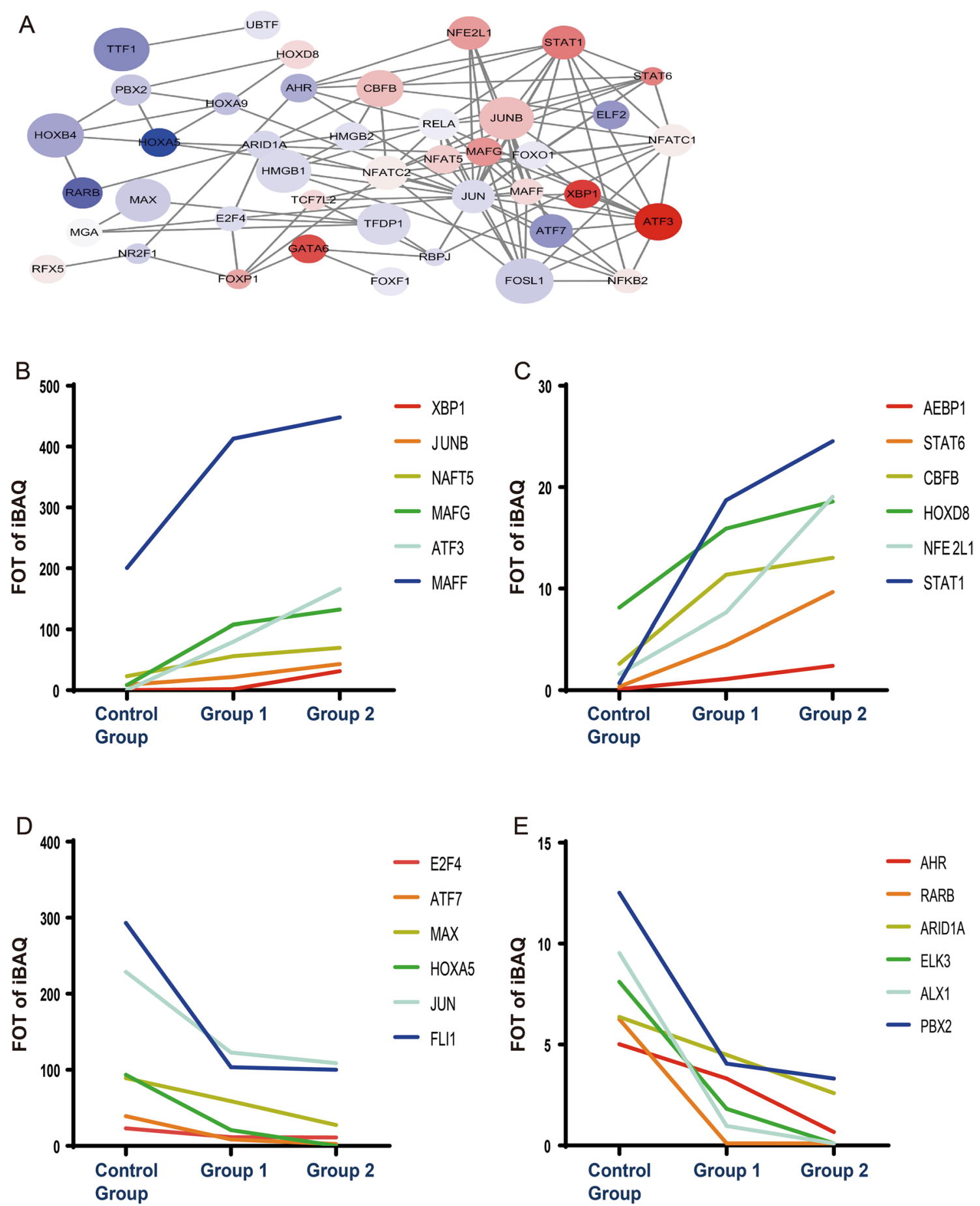

Fig. 3 Protein interaction network analysis and differential TFs with increased or decreased expression between the control group and group 2. a Relational network among the 51 transcription factors that showed significant differential expression among the three experimental groups. The area of the circle represents the number of interactions of the transcription factor. Red and blue represent upregulation and downregulation, respectively. b-e Twenty-four differential TFs increased or decreased from the control group to group 2 


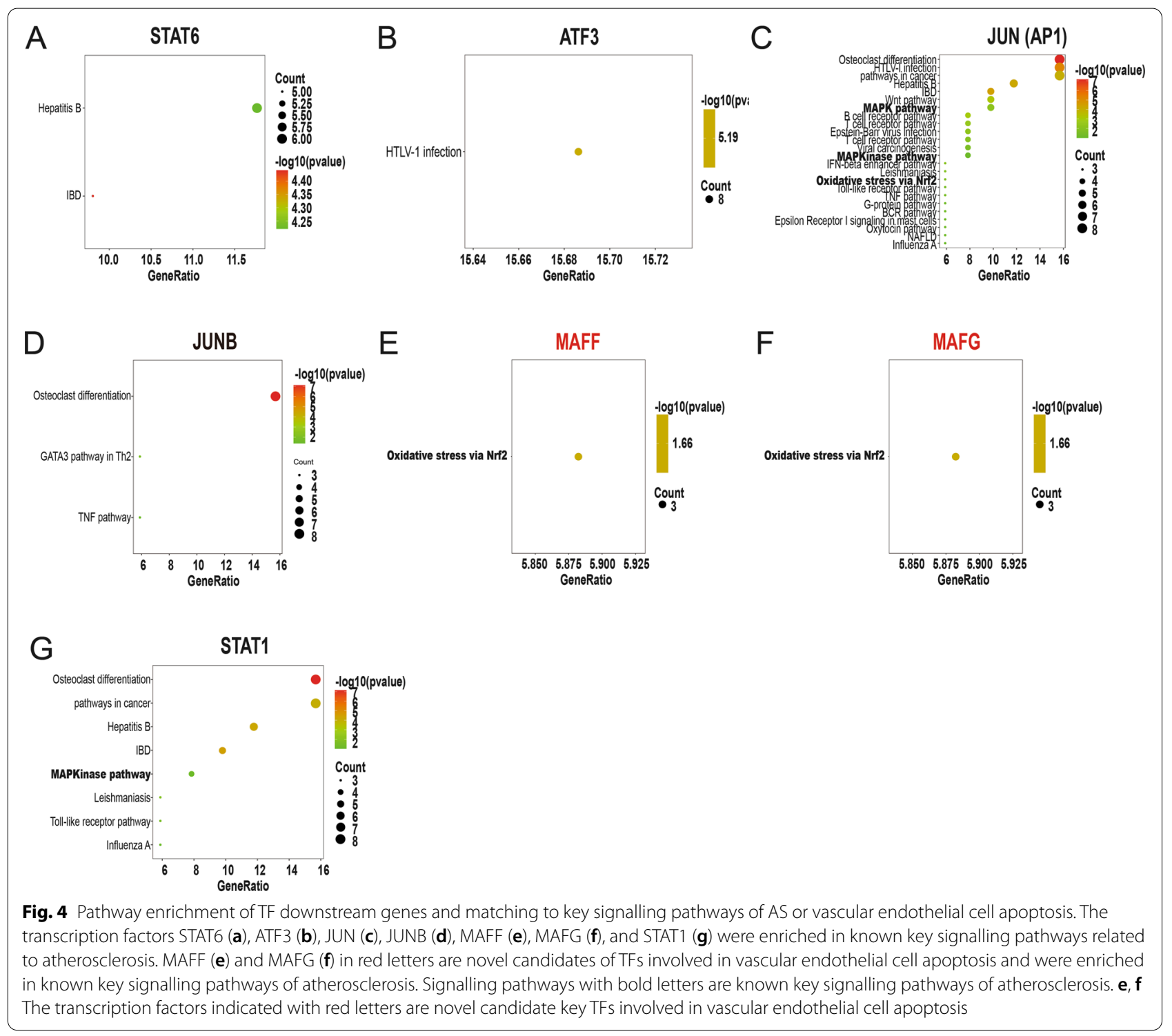

WB analysis and RT-qPCR. The results of RT-qPCR (Fig. 5a, b) and WB analysis (Fig. 5c-f) showed that the change trends in these two TFs were consistent with the change trends in the TFRE results; thus, MAFF and MAFG were verified as two novel candidate key TFs in AS.

\section{Alleviation of apoptosis induced by PA in SV40T-transformed HUVECs with MAFF and MAFG overexpression (OE)}

To validate the roles of the novel candidate TFs, we performed biological cell functionality validation experiments to reveal whether MAFF and MAFG can inhibit the PA-induced apoptosis of SV40T-transformed HUVECs. HUVECs were grouped into 4 experimental groups: the control group (no plasmid), empty plasmid group, MAFF OE group, and MAFG OE group. The RT-qPCR and WB analysis results showed that MAFF and MAFG were indeed significantly overexpressed in the MAFF OE group and MAFG OE group, respectively. The four groups were induced by $500 \mu \mathrm{M}$ PA for $24 \mathrm{~h}$. RT-qPCR and WB analysis results showed that MAFF and MAFG were overexpressed in the MAFF OE group and MAFG OE group, respectively (Fig. 6a-d). The flow cytometry results (Fig. 6e-p) showed that early apoptosis was significantly inhibited in HUVECs with MAFF OE $(30.23 \% \pm 2.26 \%$ vs $52.37 \% \pm 2.10 \%$, $P<0.001)$ or MAFG OE $(39.4 \% \pm 3.21 \%$ vs $52.37 \% \pm 2.10 \%$, $P=0.019)$ compared with HUVECs transfected with empty plasmid (Fig. 6q). 

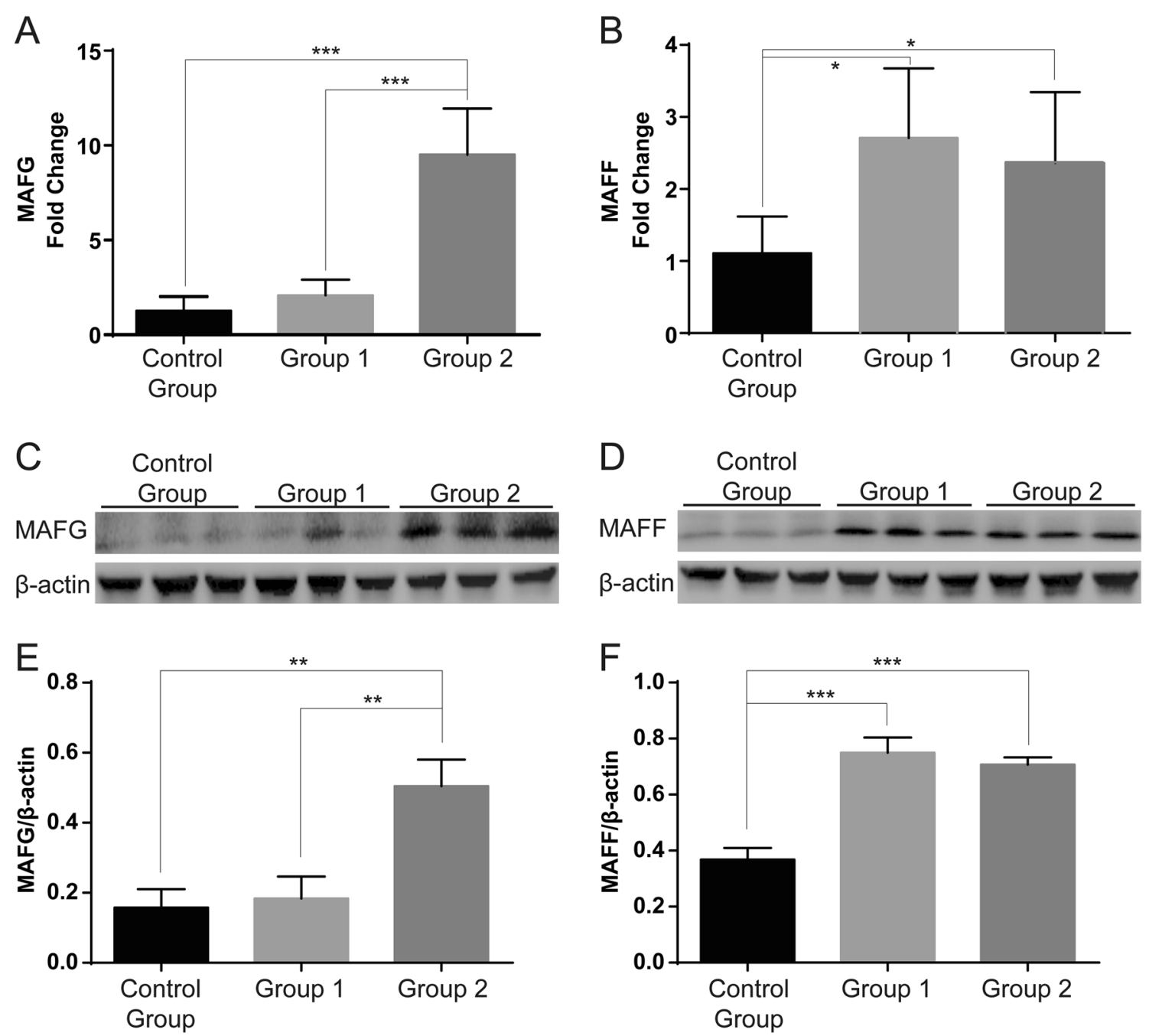

Fig. 5 WB and RT-qPCR verification of the two novel candidate key TFs, MAFF and MAFG. a, b RT-qPCR validation results. c-f WB validation results. The change trends in MAFF and MAFG in the RT-qPCR and WB analysis results were consistent with the change trends in the TFRE results, so MAFF and MAFG were validated as two novel candidate key TFs in AS. ${ }^{*}$ indicates $0.01<P<0.05$, ${ }^{* *}$ indicates $0.001<P<0.01$, and ${ }^{* * *}$ indicates $P<0.001$

\section{Discussion}

\section{Major findings}

First, we established a HUVEC apoptosis model to simulate the onset process of atherosclerosis. Then, we identified 51 TFs that showed significant differential expression with a t-test $P$ value $<0.05$ and fold change $>2$. Then, seven overlapping TFs between the significantly differentially regulated and interconnected TFs and the differentially expressed TFs between the control group and group 2 (with dose dependence) were identified, four

\footnotetext{
(See figure on next page.)

Fig. 6 Alleviation of early apoptosis induced by PA in SV40T-transformed HUVECs overexpressing MAFF and MAFG. a RT-qPCR validation of MAFF OE. $\mathbf{b}$ RT-qPCR validation of MAFG OE. c WB validation of MAFF OE. $\mathbf{d}$ WB validation of MAFG OE. $\mathbf{e}-\mathbf{h}$ The first apoptosis assay by flow cytometry. $\mathbf{i}-\mathbf{I}$ The second apoptosis assay by flow cytometry. $\mathbf{m}-\mathbf{p}$ the third time of apoptosis assay by flow cytometry. $\mathbf{e}, \mathbf{i}, \mathbf{m}$ Control-500 $\mu \mathrm{M}$ PA group subjected to three independent apoptosis assays by flow cytometry. $\mathbf{f}, \mathbf{j}, \mathbf{n}$ Plasmid-500 $\mu \mathrm{M}$ PA group subjected to three independent apoptosis assays by flow cytometry. $\mathbf{g}, \mathbf{k}$, o Results for the MAFF OE-500 $\mu \mathrm{M}$ PA group in the three independent apoptosis assays by flow cytometry. h, I, p Results for the MAFG OE-500 $\mu$ M PA group in the three independent apoptosis assays by flow cytometry. $\mathbf{q}$ Histogram summary results in the four groups. The dots located in Q4 represent early apoptotic cells. OE represents overexpression. ${ }^{* *}$ indicates $0.001<P<0.01$ and ${ }^{* * *}$ indicates $P<0.001$. Control-500 $\mu \mathrm{M}$ PA: without transfection and with $500 \mu \mathrm{M}$ PA stimulation. Plasmid-500 $\mu \mathrm{M}$ PA: with empty plasmid transfection and with $500 \mu \mathrm{M}$ PA stimulation.

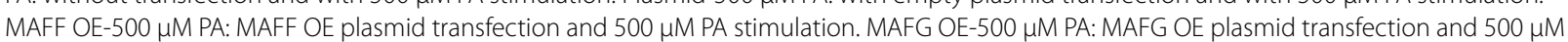
PA stimulation
} 

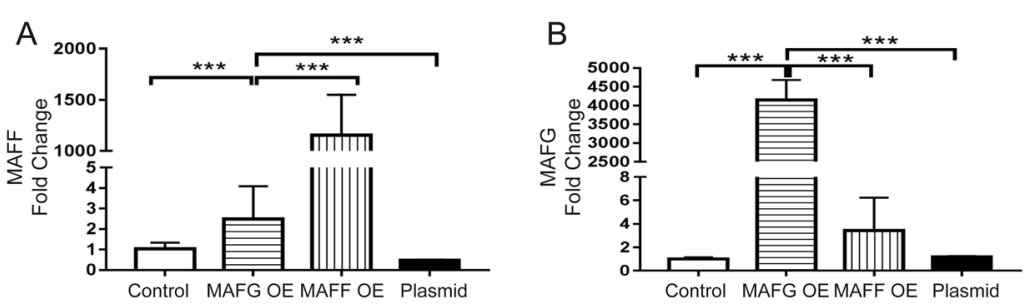

C Control Plasmid MAFF OE MAFG OE MAFF $\cdots+\cdots+$ ats $-\cdots$ $\beta$-actin $=-\infty+\cdots,-\infty--\infty--$

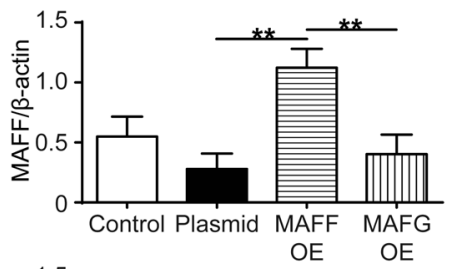

$D$
MAFG Control Plasmid MAFF OE MAFG OE $\beta$-actin $--\infty+\infty,-\infty-\infty-\infty-$
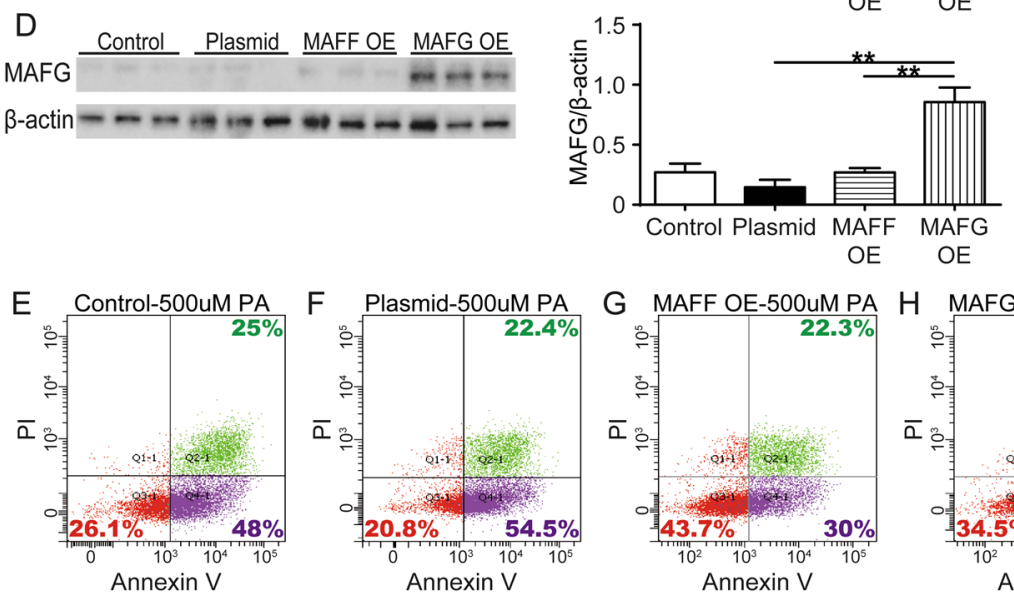

F Plasmid-500uM PA
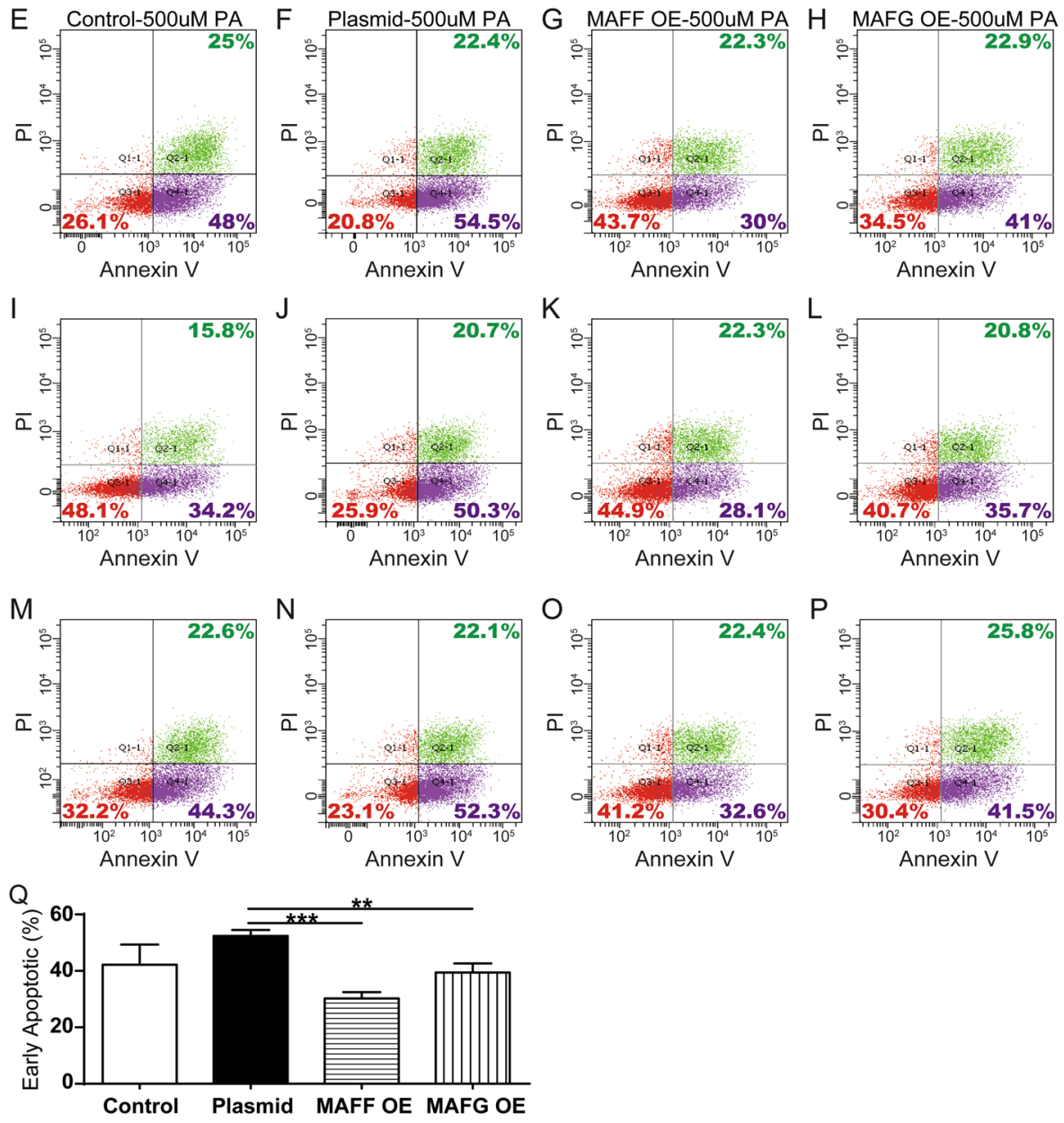

Fig. 6 (See legend on previous page.) 
of which were found to have downstream genes matching known signalling pathways of AS. The involvement of two of the four TFs-MAFF and MAFG-was still unknown in AS, and the WB and RT-qPCR verification results demonstrated that these two TFs were two novel candidate key TFs in AS. The biological cellular functionality validation results revealed that MAFF and MAFG could significantly inhibit PA-induced early apoptosis of SV40T-transformed HUVECs.

\section{Significance of this study}

Numerous TFs are involved in vascular endothelial cell apoptosis, which is the key process underlying AS onset. However, proteome-scale transcriptional profiling to identify TFs associated with this key event in AS pathogenesis has never been performed. Our results revealed two novel key TFs. These 2 novel key TFs involved in vascular endothelial cell apoptosis are probably protective TFs for AS by inhibiting apoptosis of arterial endothelial cells, and they may be useful for early-stage medical intervention in AS.

\section{Comparison with other relevant studies and deductions from major results}

Several studies have reported key TFs in AS, which were validated by observing changes in the atherosclerotic plaque area and thickness after manipulation of TF gene expression in an ApoE-mouse AS model [6, 10-27]. In this study, we also identified some of these key TFs, such as ATF3 and JUN (AP1). In addition, ALX1, ELK3, RARB, ATF7, MAFG, NFE2L1, CBFB, PBX2, MAX, FLI1, ARID1A, and MAFF were newly identified TFs reported in our present study as key TFs in AS.

According to the Genome Informatics database, all 24 known and unknown TFs identified here are involved in AS-related biological processes, such as cell proliferation and apoptosis. Our results showed that 4 candidate TFs were upregulated from the control group to group 2 in a dose-dependent manner. Considering that the concentration of PA was increased from 0 to $500 \mu \mathrm{M}$ between the control group and group 2, the upregulation of the 4 TFs in group 2 compared to the control group suggests a relevant association of these TFs with vascular endothelial cell apoptosis, making them attractive targets for early therapeutic intervention. The key signalling pathways of AS on which current research has focused are the NFE2L2-ARE, MAPK, and NFKB pathways [28, 29]. In addition, pathway enrichment for downstream genes of JUN (AP1), MAFG, MAFF, and STAT1 showed an association with key AS signalling pathways. However, JUN (AP1) and STAT1 had already been biologically validated as key TFs in AS in a previous study $[8,9]$. Therefore, we deduced from our results that MAFF and MAFG might play a key role in HUVEC apoptosis via the NFE2L2-ARE signalling pathway, because transcription factors MAFF and MAFG were done pathway enrichment to NFE2L2, which is the key molecule of the NFE2L2-ARE signalling pathway. Our results demonstrated that MAFF and MAFG are two novel key TFs in vascular endothelial cell apoptosis.

The functions of v-maf musculoaponeurotic fibrosarcoma oncogene family protein G (MAFG) and v-maf musculoaponeurotic fibrosarcoma oncogene family protein $\mathrm{F}$ (MAFF) are now known as proliferation and differentiation coactivators acting in cooperation with NFE2L2 in the mouse hepatoma cell line Hepa1c1c7 (Hepa1) via the Keap1-NFE2L2 pathway [30], and the protein MAFG acts in cooperation with NFE2L2 (Nrf2) to transcriptionally govern either repressive or activating gene functions in antioxidant response element (ARE)-dependent antioxidant pathways in skeletal muscle cells [31]. The protein MAFG is also involved in bile acid homeostasis [32], liver cancer[33], osteosarcoma[34], and central nervous system inflammation[35]. Von Scheidt M et al.[36] demonstrated that MAFF is a novel central regulator of an atherosclerosis-relevant liver network and that MAFF can trigger context-specific expression of LDLR and other genes known to affect coronary artery disease risk. Our research team has published several articles in this field demonstrating that the transcription factors STAT3 and NF-kappaB are involved in atherosclerosis pathogenesis [37-40].

However, NFE2L1 was significantly increased between the control group and experimental groups compared with NFE2L2 in our study, accompanied by a significant increase in MAFF and MAFG. Therefore, we speculate that MAFF, MAFG, and NFE2L1 can transcriptionally activate ARE-associated genes collaboratively in arterial endothelial cells, and the expression of these AREassociated genes leads to antioxidation enhancement of arterial endothelial cells. Enhancement of antioxidation and reduction in cell injury in the arterial endothelium play crucial roles in atherogenesis suppression. Here we indicated that both MAFF and MAFG could protect HUVECs from apoptosis, indicating that these two TFs might be effective targets for AS treatment.

\section{Limitations}

First, TFRE profiling is a semiquantitative method with limited accuracy. Second, the novel key TFs need to be validated experimentally in the ApoE-/- mouse AS model, and this could be achieved in a future study. 


\section{Conclusion}

Here, we identified two novel key TFs, MAFF and MAFG, involved in PA+glucose-induced vascular endothelial cell apoptosis, which is a key event underlying the onset of AS. These results need to be further biologically validated in the ApoE-/- mouse AS model in the future (Additional file 3).

\section{Abbreviations}

AS: Atherosclerosis; HUVEC: Human umbilical vein endothelial cell; PA: Palmitic acid; TFs: Transcription factors; TFRE: TF response element; LC-MS: Nano-liquid chromatography/tandem mass spectrometry; MAFG: V-maf musculoaponeurotic fibrosarcoma oncogene family protein G; MAFF: V-maf musculoaponeurotic fibrosarcoma oncogene family protein F; WB: Western blot; RT-qPCR: Real-time quantitative PCR; OE: Overexpression.

\section{Supplementary Information}

The online version contains supplementary material available at https://doi. org/10.1186/s12872-021-02246-5.

Additional file 1: Figure S1 Schematic diagram of plasmid construction. Plasmid pIRES2-EGFP containing human MAFF cDNA (pMAFF-IRES2-EGFP).

Additional file 2: Figure S2 Plasmid construction schematic diagram. Plasmid pIRES2-EGFP containing human MAFG cDNA (pMAFG-IRES2-EGFP).

Additional file 3: Figure S3 Images of original Western Blots. (A) The original Western Blots image of MAFF blots in Figure 5D. (B) The original Western Blots image of $\beta$-actin blots in Figure 5D. (C) The original Western Blots image of MAFG blots in Figure 5C. (D) The original Western Blots image of $\beta$-actin blots in Figure $5 C$. (E) The original Western Blots image of MAFF blots in Figure 6C. (F) The original Western Blots image of MAFG blots in Figure 6D. $(G)$ The original Western Blots image of $\beta$-actin blots in Figure 6C and 6D.

Additional file 4: Table S1 TFRE results. Significantly different transcription factors among the control group, group 1, and group 2 .

Additional file 5: Table S2 TFRE results. Significantly different transcription factors between group 1 and the control group met the selection criteria of both a $P$ value $<0.05$ and a fold change $>2$

Additional file 6: Table S3 TFRE results. Significantly different transcription factors between group 2 and group 1 met the selection criteria of both $P$ value $<0.05$ and fold change $>2$

Additional file 7: Table S4 TFRE results. Significantly different transcription factors between group 2 and the control group met the selection criteria of both a $P$ value $<0.05$ and a fold change $>2$

Additional file 8: Raw TFRE data. Raw proteome-scale profiling data.

\section{Acknowledgements}

We would like to thank Dr. Lei Song and Mingwei Liu for their support with the TFRE experiments.

\section{Authors' contributions}

MW designed the study; performed the TFRE experiments, TFRE data analysis and cellular functional validation experiments; and wrote the manuscript. FL performed HUVEC culture and established the apoptotic cell model of palmitic acid-induced atherosclerosis. BF performed the WB and RT-qPCR experiments. $\mathrm{QH}$ and $Y Y$ designed the study. All authors have read and approved the manuscript.

\section{Funding}

This work was supported by the Youth Funds of the Natural Science Foundation of Xinjiang Uygur Autonomous Region (2020D01C256) and the Funds of the Tianshan Cedar Project Foundation of Xinjiang Uygur Autonomous Region (2020XS29). These funding bodies did not play any role in the design of the study; collection, analysis, or interpretation of the data; or manuscript writing.

\section{Availability of data and materials}

The first author can be contacted if the raw data are needed. The email address of the first author is wangmangyuan2013@163.com.

\section{Declarations}

Ethics approval and consent to participate

Not applicable.

\section{Consent to publication}

Not applicable.

\section{Competing interests}

The authors declare that they have no competing interests.

\section{Author details}

${ }^{1}$ Clinical Medicine Postdoctoral Research Station, The First Affiliated Hospital of Xinjiang Medical University, 137, Liyushan Road, Xin Shi District, Urumqi 830054, People's Republic of China. ${ }^{2}$ Department of Cardiac Surgery, The First Affiliated Hospital of Xinjiang Medical University, 137, Liyushan Road, Xin Shi District, Urumqi 830054, People's Republic of China. ${ }^{3}$ Department of Cardiology, The First Affiliated Hospital of Xinjiang Medical University, 137, Liyushan Road, Xin Shi District, Urumqi 830054, People's Republic of China.

${ }^{4}$ Xinjiang Key Laboratory of Cardiovascular Disease Research, Urumqi, People's Republic of China.

Received: 15 September 2020 Accepted: 8 September 2021

Published online: 17 September 2021

\section{References}

1. Hopkins PN. Molecular biology of atherosclerosis. Physiol Rev. 2013;93(3):1317-542.

2. Benjamin EJ, Virani SS, Callaway CW, Chamberlain AM, Chang AR, Cheng S, Chiuve SE, Cushman M, Delling FN, Deo R, et al. Heart disease and stroke statistics-2018 update: a report from the American Heart Association. Circulation. 2018;137(12):e67-492.

3. Dagher Z, Ruderman N, Tornheim K, Ido Y. Acute regulation of fatty acid oxidation and amp-activated protein kinase in human umbilical vein endothelial cells. Circ Res. 2001;88(12):1276-82.

4. Marin EP, Derakhshan B, Lam TT, Davalos A, Sessa WC. Endothelial cell palmitoylproteomic identifies novel lipid-modified targets and potential substrates for protein acyl transferases. Circ Res. 2012;110(10):1336-44.

5. Ding C, Chan DW, Liu W, Liu M, Li D, Song L, Li C, Jin J, Malovannaya A, Jung SY, et al. Proteome-wide profiling of activated transcription factors with a concatenated tandem array of transcription factor response elements. Proc Natl Acad Sci USA. 2013;110(17):6771-6.

6. Niu N, Xu S, Xu Y, Little PJ, Jin ZG. Targeting mechanosensitive transcription factors in atherosclerosis. Trends Pharmacol Sci. 2019;40(4):253-66.

7. Wang M, Hu S, Nie Y, Song J. Proteomic profiling of key transcription factors in the process of neonatal mouse cardiac regeneration capacity loss. Cell Biol Int. 2019

8. Partridge J, Carlsen H, Enesa K, Chaudhury H, Zakkar M, Luong L, Kinderlerer A, Johns M, Blomhoff R, Mason JC, et al. Laminar shear stress acts as a switch to regulate divergent functions of NF-kappaB in endothelial cells. FASEB J Off Publ Fed Am Soc Exp Biol. 2007;21(13):3553-61.

9. Wang Y, Han Z, Fan Y, Zhang J, Chen K, Gao L, Zeng H, Cao J, Wang C. MicroRNA-9 Inhibits NLRP3 inflammasome activation in human atherosclerosis inflammation cell models through the JAK1/STAT signaling pathway. Cell Physiol Biochem Int J Exp Cell Physiol Biochem Pharmacol. 2017:41(4):1555-71.

10. Gold ES, Ramsey SA, Sartain MJ, Selinummi J, Podolsky I, Rodriguez DJ, Moritz RL, Aderem A. ATF3 protects against atherosclerosis by suppressing 25-hydroxycholesterol-induced lipid body formation. J Exp Med. 2012;209(4):807-17. 
11. Nazari-Jahantigh M, Wei Y, Noels H, Akhtar S, Zhou Z, Koenen RR, Heyll K, Gremse F, Kiessling F, Grommes J, et al. MicroRNA-155 promotes atherosclerosis by repressing Bcl6 in macrophages. J Clin Investig. 2012;122(11):4190-202.

12. Joly AL, Seitz C, Liu S, Kuznetsov NV, Gertow K, Westerberg LS, PaulssonBerne G, Hansson GK, Andersson J. Alternative splicing of FOXP3 controls regulatory $T$ cell effector functions and is associated with human atherosclerotic plaque stability. Circ Res. 2018;122(10):1385-94.

13. Li K, Ching D, Luk FS, Raffai RL. Apolipoprotein E enhances microRNA146 a in monocytes and macrophages to suppress nuclear factor-kappaBdriven inflammation and atherosclerosis. Circ Res. 2015;117(1):e1-11.

14. Chen HH, Keyhanian K, Zhou X, Vilmundarson RO, Almontashiri NA, Cruz SA, Pandey NR, Lerma Yap N, Ho T, Stewart CA, et al. IRF2BP2 reduces macrophage inflammation and susceptibility to atherosclerosis. Circ Res. 2015;117(8):671-83.

15. Sayin VI, Khan OM, Pehlivanoglu LE, Staffas A, Ibrahim MX, Asplund A, Agren P, Nilton A, Bergstrom G, Bergo MO, et al. Loss of one copy of Zfp148 reduces lesional macrophage proliferation and atherosclerosis in mice by activating p53. Circ Res. 2014;115(9):781-9.

16. Guo Y, Fan Y, Zhang J, Lomberk GA, Zhou Z, Sun L, Mathison AJ, GarciaBarrio MT, Zhang J, Zeng L, et al. Perhexiline activates KLF14 and reduces atherosclerosis by modulating ApoA-I production. J Clin Investig. 2015;125(10):3819-30

17. Hsieh J, Koseki M, Molusky MM, Yakushiji E, Ichi I, Westerterp M, Iqbal J, Chan RB, Abramowicz S, Tascau L, et al. TTC39B deficiency stabilizes $L X R$ reducing both atherosclerosis and steatohepatitis. Nature. 2016;535(7611):303-7.

18. Gage MC, Becares N, Louie R, Waddington KE, Zhang Y, Tittanegro TH, Rodriguez-Lorenzo S, Jathanna A, Pourcet B, Pello OM, et al. Disrupting LXRalpha phosphorylation promotes FoxM1 expression and modulates atherosclerosis by inducing macrophage proliferation. Proc Natl Acad Sci USA. 2018;115(28):E6556-e6565.

19. Zhuang T, Liu J, Chen X, Zhang L, Pi J, Sun H, Li L, Bauer RC, Wang H, Yu $Z$, et al. Endothelial Foxp1 suppresses atherosclerosis via modulation of Nirp3 inflammasome activation. Circ Res. 2019.

20. Mahmoud MM, Kim HR, Xing R, Hsiao S, Mammoto A, Chen J, SerbanovicCanic J, Feng S, Bowden NP, Maguire R, et al. TWIST1 integrates endothelial responses to flow in vascular dysfunction and atherosclerosis. Circ Res. 2016;119(3):450-62.

21. Dunn J, Qiu H, Kim S, Jjingo D, Hoffman R, Kim CW, Jang I, Son DJ, Kim $D$, Pan C, et al. Flow-dependent epigenetic DNA methylation regulates endothelial gene expression and atherosclerosis. J Clin Investig. 2014;124(7):3187-99.

22. Sage AP, Nus M, Bagchi Chakraborty J, Tsiantoulas D, Newland SA, Finigan AJ, Masters L, Binder CJ, Mallat Z. X-box binding protein-1 dependent plasma cell responses limit the development of atherosclerosis. Circ Res. 2017;121(3):270-81.

23. Lim WS, Timmins JM, Seimon TA, Sadler A, Kolodgie FD, Virmani R, Tabas I. Signal transducer and activator of transcription-1 is critical for apoptosis in macrophages subjected to endoplasmic reticulum stress in vitro and in advanced atherosclerotic lesions in vivo. Circulation. 2008;117(7):940-51.

24. Xu L, Cheng D, Huang Z, Ding S, Zhang W, Tan H, Shi H, Chen R, Zou Y, Wang TC, et al. Histamine promotes the differentiation of macrophages from CD11b(+) myeloid cells and formation of foam cells through a Stat6-dependent pathway. Atherosclerosis. 2017;263:42-52.

25. Majdalawieh A, Zhang L, Fuki IV, Rader DJ, Ro HS. Adipocyte enhancerbinding protein 1 is a potential novel atherogenic factor involved in macrophage cholesterol homeostasis and inflammation. Proc Natl Acad Sci USA. 2006;103(7):2346-51.
26. Bock KW. Human AHR functions in vascular tissue: pro- and anti-inflammatory responses of AHR agonists in atherosclerosis. Biochem Pharmacol. 2019;159:116-20

27. Halterman JA, Kwon HM, Leitinger N, Wamhoff BR. NFAT5 expression in bone marrow-derived cells enhances atherosclerosis and drives macrophage migration. Front Physiol. 2012;3:313.

28. Pan JX. LncRNA H19 promotes atherosclerosis by regulating MAPK and NF-kB signaling pathway. Eur Rev Med Pharmacol Sci. 2017;21(2):322-8.

29. Chen $B$, Lu Y, Chen Y, Cheng J. The role of Nrf2 in oxidative stress-induced endothelial injuries. J Endocrinol. 2015;225(3):R83-99.

30. Hirotsu Y, Katsuoka F, Funayama R, Nagashima T, Nishida Y, Nakayama K, Engel JD, Yamamoto M. Nrf2-MafG heterodimers contribute globally to antioxidant and metabolic networks. Nucl Acids Res. 2012;40(20):10228-39.

31. Caggiano R, Cattaneo F, Moltedo O, Esposito G, Perrino C, Trimarco B, Ammendola R, Faraonio R. miR-128 is implicated in stress responses by targeting MAFG in skeletal muscle cells. Oxid Med Cell Longev. 2017:2017:9308310

32. de Aguiar Vallim TQ, Tarling EJ, Ahn H, Hagey LR, Romanoski CE, Lee RG, Graham MJ, Motohashi H, Yamamoto M, Edwards PA. MAFG is a transcriptional repressor of bile acid synthesis and metabolism. Cell Metab. 2015;21(2):298-311.

33. Liu T, Yang H, Fan W, Tu J, Li TWH, Wang J, Shen H, Yang J, Xiong T, Steggerda J, et al. Mechanisms of MAFG dysregulation in cholestatic liver injury and development of liver cancer. Gastroenterology. 2018;155(2):557-571. e514.

34. Shan HJ, Zhu LQ, Yao C, Zhang ZQ, Liu YY, Jiang Q, Zhou XZ, Wang XD, Cao C. MAFG-driven osteosarcoma cell progression is inhibited by a novel miRNA miR-4660. Mol Ther Nucl Acids. 2021;24:385-402.

35. Wheeler MA, Clark IC, Tjon EC, Li Z, Zandee SEJ, Couturier CP, Watson BR, Scalisi G, Alkwai S, Rothhammer V, et al. MAFG-driven astrocytes promote CNS inflammation. Nature. 2020;578(7796):593-9.

36. von Scheidt M, Zhao Y, de Aguiar Vallim TQ, Che N, Wierer M, Seldin MM Franzén O, Kurt Z, Pang S, Bongiovanni D, et al. Transcription factor MAFF (MAF Basic Leucine Zipper Transcription Factor F) regulates an atherosclerosis relevant network connecting inflammation and cholesterol metabolism. Circulation. 2021;143(18):1809-23.

37. Li X, Ma YT, Xie X, Yang YN, Ma X, Zheng YY, Pan S, Liu F, Chen BD. Association of Egr3 genetic polymorphisms and coronary artery disease in the Uygur and Han of China. Lipids Health Dis. 2014;13:84.

38. Maimaiti A, Maimaiti A, Yang Y, Ma Y. MiR-106b exhibits an anti-angiogenic function by inhibiting STAT3 expression in endothelial cells. Lipids Health Dis. 2016;15:51.

39. Wang X, Chen Q, Pu H, Wei Q, Duan M, Zhang C, Jiang T, Shou X, Zhang J, Yang Y. Adiponectin improves NF-kB-mediated inflammation and abates atherosclerosis progression in apolipoprotein E-deficient mice. Lipids Health Dis. 2016;15:33.

40. Zhai H, Chen QJ, Gao XM, Ma YT, Chen BD, Yu ZX, Li XM, Liu F, Xiang Y, Xie J, et al. Inhibition of the NF-kB pathway by R65 ribozyme gene via adeno-associated virus serotype 9 ameliorated oxidized LDL induced human umbilical vein endothelial cell injury. Int J Clin Exp Pathol. 2015;8(9):9912-21.

\section{Publisher's Note}

Springer Nature remains neutral with regard to jurisdictional claims in published maps and institutional affiliations. 